\title{
On bounded analytic functions and closure problems
}

\author{
By Lennart Carleson
}

\section{Introduction}

1. Let us denote by $H^{p}, p \geq 1$, the space of functions $f(z)$ holomorphic in $|z|<1$ and such that

$$
N_{p}(f)=\lim _{r \rightarrow 1}\left\{\frac{1}{2 \pi} \int_{0}^{2 \pi}\left|f\left(r e^{i \theta}\right)\right|^{p} d \theta\right\}^{\frac{1}{p}}=\left\{\frac{1}{2 \pi} \int_{0}^{2 \pi}\left|f\left(e^{i \theta}\right)\right|^{p} d \theta^{\frac{1}{p}}<\infty,\right.
$$

where $f\left(e^{i \theta}\right)=\lim _{r \rightarrow 1} f\left(r e^{i \theta}\right)$ a. e. It is obvious that $H^{p}$ is a Banach space under the norm $N_{p}$. If we combine the wellknown representation of a linear functional on $L^{p}(0,2 \pi)$ with a theorem of M. Riesz on conjugate functions, we find that the general linear functional on $H^{p}, p>1$, has the form

$$
L(f)=\int_{0}^{2 \pi} f\left(e^{i \theta}\right) \overline{g\left(e^{i \theta}\right)} d \theta, \quad g \in H^{q}, \quad p^{-1}+q^{-1}=1 .
$$

The simple structure of the general linear functional on $H^{p}$ is the key to a great number of results for these spaces.

The "limit space" as $p \rightarrow \infty$ is the space $B$ of bounded analytic functions in $|z|<1$ with the uniform norm

$$
\|f\|=\sup _{|z|<1}|f(z)| .
$$

Although this space has a simpler function-theoretic nature than $H^{p}$, its theory as a Banachspace is extremely complicated. This fact depends to a great extent on the absence of a simple representation for linear functionals. On the other hand, $B$ is not only a Banach space, but also a Banach algebra.

If one seeks results for $B$ which for $H^{p}$ depend on the formula (1), the following question should be asked: how shall we weaken the norm (2) in $B$ in order to ensure that the functionals have a representation of type (1)? In the first section we shall treat this problem by introducing certain weight functions. The method will also be used to find a function-theoretic correspondance to weak convergence on a finite interval. 
L. CARLESON, On bounded analytic functions and closure problems

In the second section, we shall consider a closure problem for $B$ where the relation between $B$ and its subring $C$ of uniformly continuous functions will be of importance. Finally, we shall make an application of the results to the PickNevanlinna interpolation problem.

\section{Section I}

2. When we look for functionals on $B$ analogous to (1), there are two essentially different possibilities: we may take for $g$ a function in $H^{1}$ or in $L^{1}(0,2 \pi)$. These representations are fundamentally different since the above-mentioned theorem on conjugate functions fails for $p=1$. Let us call the two types of representations $(A)$ and $(B)$ :

$$
\begin{aligned}
& L(f)=\int_{0}^{2 \pi} f\left(e^{i \theta}\right) \overline{g\left(e^{i \theta}\right)} d \theta, \quad g \in H^{1} \\
& L(f)=\int_{0}^{2 \pi} f\left(e^{i \theta}\right) K(\theta) d \theta, \quad K \in L^{1}(0,2 \pi) .
\end{aligned}
$$

In the sequel, let $\mu(r)$ denote a continuous function on $0 \leq r \leq 1$ such that $0 \leq \mu(r) \leq 1$ and $\mu(1)=0$. Furthermore, let $C_{\mu}$ be the space of functions analytic in $|z|<1$ such that $\lim _{r \rightarrow 1} f\left(r e^{i \theta}\right) \mu(r)=0$ uniformly in $\theta$. If we introduce the norm

$$
\|f\|_{\mu}=\sup _{r<1} \mu(r)\left|f\left(r e^{i \theta}\right)\right|,
$$

$C_{\mu}$ becomes a Banach space.

By means of the weight functions $\mu$, we can now solve our problem. Let us first consider functionals of type (A).

Theorem 1. Every linear functional on $C_{\mu}$ has on its subspace $B$ a representation of the form $(A)$ if and only if

$$
\varlimsup_{r \rightarrow 1} \mu(r) \log \frac{1}{1-r}<\infty
$$

Let us first assume that condition (3) is satisfied, and let $L(f)$ be a linear functional on $C_{\mu}$. By a theorem of Riesz-Banach, there exists a function $\sigma(z)$ of bounded variation in $|z|<1$ such that

$$
L(f)=\iint_{|z|<1} \mu(|z|) f(z) d \sigma(z), \quad f \in C_{\mu} .
$$

If now $f \in B$ and is represented by its Cauchy integral, we find that if

$$
g_{\ell}(\zeta)=\frac{1}{2 \pi} \int_{|z|<\ell} \int_{\mid<} \frac{\mu(|z|)}{1-\bar{z} \zeta} d \overline{\sigma(z)}
$$

and if $L_{\varrho}(f)$ is the functional of type (A) defined by this function, then $\lim _{\varrho \rightarrow 1} L_{\varrho}(f)=L(f)$. It follows for $\varrho<\varrho^{\prime}<1$ 


$$
\varlimsup_{\varrho, \varrho^{\prime} \rightarrow 1} N_{1}\left(g_{Q}-g_{Q^{\prime}}\right) \leqq \text { Const. } \sup _{r<1} \mu(r) \log \frac{1}{1-r} \varlimsup_{\varrho, \varrho^{\prime}+1} \int_{\varrho \leq\left\{|z| \leq \varrho^{\prime}\right.}|d \sigma(z)|=0 .
$$

Hence $g \in H^{1}$ exists such that $N_{1}\left(g-g_{\varrho}\right) \rightarrow 0, \varrho \rightarrow 1$, and the functional of type (A) defined by this function $g$ coincides with $L$ on the space $B$. The first part of the theorem is eonsequently proved.

If, on the other hand, (3) does not hold, there exists a sequence $\left\{r_{v}\right\}_{1}^{\infty}, r_{v} \uparrow 1$, such that

$$
\mu_{\nu}=\mu\left(r_{\nu}\right) \log \frac{1}{1-r_{\nu}} \rightarrow \infty, \quad \nu \rightarrow \infty .
$$

We then form the following expression, which is easily seen to be a linear functional on $C_{\mu}$ :

$$
L(f)=\sum_{\nu=1}^{\infty} f\left(r_{\nu}\right) \mu\left(r_{\nu}\right) \lambda_{\nu} .
$$

$\left\{\lambda_{v}\right\}_{1}^{\infty}$ is here a sequence of positive numbers such that

$$
\sum_{1}^{\infty} \lambda_{v}<\infty \text { and } \sum_{v=1}^{\infty} \lambda_{v} \mu_{v}=\infty
$$

For $f$ belonging to $B$, we have $L(f)=\lim _{n \rightarrow \infty} L_{n}(f)$, where $L_{n}$ is the functional of type (A) defined by the function

$$
g_{n}(z)=\frac{1}{2 \pi} \sum_{\nu=1}^{n} \frac{\lambda_{\nu} \mu\left(r_{\nu}\right)}{1-r_{v} z}
$$

Let us now assume that the functional defined above has a representation of type (A) on $B$. If $h(\theta)$ is an arbitrary function with continuous derivative and period $2 \pi$, then

$$
\lim _{n \rightarrow \infty} \int_{0}^{2 \pi} h(\theta) \overline{g_{n}\left(e^{i \theta}\right)} d \theta=\int_{0}^{2 \pi} h(\theta) \overline{g\left(e^{i \theta}\right)} d \theta .
$$

Namely, if $\bar{h}(\theta)$ is the conjugate function of $h$, neither side changes its value if we add ih to $h$. But for $h+i \hbar$, (4) holds by assumption. For $\delta>0$, we choose a non-negative periodic function $h_{\delta}(\theta)$ with continuous derivative such that $h_{\delta}=0$ for $-c<\theta<0, h_{\delta} \leq 1$ for all $\theta$ and $h_{\delta}=1$ for $\delta \leq \theta<c<\pi$. By (4), we have

$$
\varlimsup_{\delta \rightarrow 0}\left|\lim _{n \rightarrow \infty} \int_{0}^{2 \pi} h_{\delta}(\theta) \overline{g_{n}\left(e^{i \theta}\right)} d \theta\right|<\infty .
$$

Going back to the expression for $g_{n}$, however, we find

$$
\begin{gathered}
\varlimsup_{\delta \rightarrow 0}\left|\lim _{n \rightarrow \infty} \operatorname{Im}\left\{\int_{0}^{2 \pi} h_{\delta}(\theta) \overline{g_{n}\left(e^{i \theta}\right)} d \theta\right\}\right| \geqq \\
\geqq \text { Const. } \lim _{\delta \rightarrow 0} \sum_{\nu=1}^{\infty} \lambda_{\nu} \mu\left(r_{\nu}\right) \int_{\delta}^{\pi} \frac{\theta d \theta}{\left|1-r_{\nu} \theta\right|^{2}} \geqq \text { Const. } \sum_{I}^{\infty} \lambda_{\nu} \mu_{\nu}=\infty .
\end{gathered}
$$




\section{CARLESON, On bounded analytic functions and closure problems}

This contradiction proves the theorem.

3. It is very easy to see - in the same way as above, if we represent the functions in $B$ by Poisson's integral instead of Cauchy's - that the following theorem is true for functionals of type $(B)$.

Theorem 2. The functionals on $C_{\mu}$ have a representation of type $(B)$ on $B$ for every choice of the weight function $\mu$.

This theorem is a particular case of a more general result which we shall now briefly discuss.

Let $D$ be the Banach space of bounded functions $\varphi(x)$ on $(0,2 \pi)$, where we have introduced the uniform norm $\|\varphi\|$. With every function in $D$, we associate the corresponding harmonic function in the unit circle

$$
u_{\varphi}=u(z ; \varphi)=\frac{1}{2 \pi} \int_{0}^{2 \pi} \frac{1-r^{2}}{1+r^{2}-2 r \cos (\theta-x)} \varphi(x) d x, \quad z=r e^{i \theta} .
$$

Let $D^{*}$ be the space of these functions $u$.

Suppose now that $S$ is a linear subset of $D$ and that $S^{*}$ is the corresponding subset of $D^{*}$. If $\bar{S}$ is the weak closure of $S$, the following theorem can be proved.

Theorem 3. A function $\psi(x) \in D$ belongs to $\bar{S}$ if and only if, for every weight function $\mu(r)$ and every $\varepsilon>0$, a function $\varphi \in S$ exists such that

$$
|u(z ; \psi)-u(z ; \varphi)|<\varepsilon \mu(|z|)^{-1}, \quad 0 \leq|z|<1 .
$$

Let us first assume that the above approximation is impossible for some $\mu(r)$. Then $u_{\psi}$ does not belong to the closure of $S^{*}$ in the metric of $D_{\mu}^{*}$, where $D_{\mu}^{*}$ is formed by harmonic functions in the same way as $C_{\mu}$ was formed by analytic functions. We conclude that a functional $L^{*}$ on $D_{\mu}^{*}$ exists which vanishes on $S^{*}$ and does not vanish for $u_{\psi}$. As before, we have a representation on $D^{*}$

$$
L^{*}\left(u_{\lambda}\right)=\iint_{|z|<1} u(z ; \lambda) \mu(|z|) d \sigma(z)
$$

where $\sigma$ is of bounded variation in $|z|<1$. If we insert the Poisson integral for $u_{\lambda}$ and change the order of integration, we get

$$
L^{*}\left(u_{\lambda}\right)=\int_{0}^{2 \pi} \lambda(x) K(x) d x
$$

where $K(x)$ belongs to $L^{1}(0,2 \pi)$. Hence $\psi$ does not belong to $\bar{S}$.

In the proof of the converse we shall use the following lemmas.

Lemma 1. If $K(x)$ belongs to $L^{1}(0,2 \pi)$ and

$$
K(x) \sim \frac{a_{0}}{2}+\sum_{1}^{\infty}\left(a_{n} \cos n x+b_{n} \sin n x\right)
$$


there exists a sequence of positive numbers $A_{n}$ with $\lim _{n \rightarrow \infty} A_{n}=\infty$, such that for every sequence $\left\{\lambda_{n}\right\}_{1}^{\infty}$ of positive numbers which is increasing, concave and satisfies $\lambda_{n} \leq A_{n}$

$$
\frac{a_{0}}{2}+\sum_{1}^{\infty} \lambda_{n}\left(a_{n} \cos n x+b_{n} \sin n x\right)
$$

is a Fourier-Stieltjes series.

Lemma 2. Given a sequence of positive numbers $a_{n}, \lim _{n \rightarrow \infty} a_{n}=0$, there exists $a$ non-negative function $h(t)$ in $L^{1}(0,1)$ such that

$$
a_{n} \leq b_{n}=\int_{0}^{1} t^{n} h(t) d t
$$

$h(t)$ can furthermore be chosen so that $\left\{b_{n}^{-1}\right\}$ is a concave sequence.

To prove lemma 1, we need only observe that the Cesaro mean of (7) converges in mean to $K(x)$ and make repeated use of partial summations in the series (7) and (8). The proof of lemma 2 is completely straight forward.

We return to the proof of theorem 3 and assume that there exists a function $K(x)$ in $L^{1}(0,2 \pi)$ so that

$$
\int_{0}^{2 \pi} K(x) \varphi(x) d x=0, \quad \varphi \in S,
$$

while the corresponding integral for $\psi$ is different from zero. From lemimas 1 and 2 we deduce that $K(x)$ has a representation

$$
K(x)=\underset{e^{* 1}}{\stackrel{(1)}{\text { i. }}} \int_{0}^{\varrho} h(r) d r \int_{0}^{2 \pi} \frac{1-r^{2}}{1+r^{2}-2 r \cos (x-\theta)} d \tau(\theta),
$$

where $\tau$ is of bounded variation and $h(r)$ belongs to $L^{1}(0,1)$. A weight function $\mu(r)$ can now be chosen so that $f(r)=h(r) \mu(r)^{-1}$ belongs to $L^{1}(0,1)$. If we define $\sigma$ by $d \sigma(z)=d \tau(\theta) f(r) d r, z=r e^{i \theta}$, it follows by absolute convergence from our assumption on $K$ that

$$
L^{*}\left(u_{\varphi}\right)=\iint_{|z|<1} u(z ; \varphi) \mu(|z|) d \sigma(z)=0, \quad \varphi \in S,
$$

while $L^{*}\left(u_{\varphi}\right) \neq 0$. This means that the approximation (5) is not possible for the weight function we have just defined. The proof of theorem 3 is thus complete.

As an illustration of the significance of the functionals (A), let us mention the following result: if $\left|a_{v}\right|<1$ and $\sum_{1}^{\infty}\left(1-\left|a_{v}\right|\right)$ diverges, then for every bounded analytic function $f(z)$ in $|z|<1$ and every $\varepsilon>0$ constants $\left\{c_{\nu}\right\}_{1}^{n}$ exist so that

$$
\left|\sum_{1}^{n} c_{v} \frac{a_{v}-z}{1-z \bar{a}_{v}}-f(z)\right|<\varepsilon \log \frac{1}{1-|z|}, \quad 0 \leq|z|<1 .
$$




\section{Section II}

4. We shall in this section study a problem which is connected with the fact that we can multiply two elements in $B$, i.e. that $B$ is a Banach algebra. It should be stressed that some of our results are easy consequences of a general result on Banach algebras - this is in particular true of theorem 5 - but it is necessary for applications to have proofs of classical nature. ${ }^{1}$

We start with the following closure theorem for the subspace $C$ of $B$ of uniformly continuous functions.

Theorom 4. If $f_{1}, f_{2}, \ldots, f_{n}$ belong to $C$, then $\left\{z^{k} f_{m}(z)\right\}, m=1,2, \ldots$, $n ; k=0,1, \ldots$, is fundamental on $C$ if and only if

$$
\left|f_{1}(z)\right|+\left|f_{2}(z)\right|+\cdots+\left|f_{n}(z)\right| \neq 0, \quad|z| \leq 1
$$

We shall prove the theorem in the case $n=2$; the general case is treated quite similarly.

Let $L(f)$ be a linear functional on $C$. which vanishes on the subspace $E$ spanned by the given functions. The representation

$$
L(f)=\int_{0}^{2 \pi} f\left(e^{i \theta}\right) d \mu(\theta)
$$

follows from the corresponding result for the space of continuous functions on $(0,2 \pi)$ without difficulty, since the spaces are separable. We thus have

$$
\begin{aligned}
& \int_{0}^{2 \pi} e^{i k \theta} f_{1}\left(e^{i \theta}\right) d \mu(\theta)=\int_{0}^{\pi} e^{i k \theta} d \mu_{1}(\theta)=0 \\
& \int_{0}^{2 \pi} e^{i k \theta} f_{2}\left(e^{i \theta}\right) d \mu(\theta)=\int_{0}^{2 \pi} e^{i k \theta} d \mu_{2}(\theta)=0
\end{aligned}
$$

From these relations it follows by a theorem of F. and M. RIEsz that $\mu_{1}$ and $\mu_{2}$ are absolutely continuous functions. We then immediately infer from our assumption (9) that also $\mu(\theta)$ is absolutely continuous. Hence $K(\theta) \in L^{1}(0,2 \pi)$ exists so that

$$
\int_{0}^{2 \pi} K(\theta) f_{m}\left(e^{i \theta}\right) e^{i k \theta} d \theta=0, \quad m=1,2 ; \quad k=0,1, \ldots{ }^{3}
$$

This means that $K(\theta) f_{m}\left(e^{i \theta}\right)$ is the boundary function of an analytic function $F_{m}(z)$ which belongs to $H^{1}$ and satisfies $F_{m}(0)=0$. Furthermore,

$$
K(\theta)=\lim _{r \rightarrow 1} \frac{F_{m}\left(r e^{i \theta}\right)}{f_{m}\left(r e^{i \theta}\right)} \text { a.e., } m=1,2 .
$$

\footnotetext{
1 See I. Gelfand and G. SrLov: Über verschiedene Methoden der Einführung der Topologie in die Menge der maximalen Ideale eines normierten Ringes. Mat. Sbornik 9 (1941).

${ }^{2}$ See e.g. Zygmunn: Trigonometrical series, Warszawa-Lwów, 1935, p. 158.

3 For the following, see Betrling, A.: On two problems concerning linear transformations in Hilbert space. Acta Math. 81 (1949).
} 
It follows that $F_{1}(z) / f_{1}(z)$ and $F_{2}(z) / f_{2}(z)$ in $|z|<1$ are two different representations of one and the same meromorphic function $H(z)$. By assumption (9), $H(z)$ is holomorphic and belongs to $H^{1}$ and its boundary function coincides a.e. with $K(\theta)$. For an arbitrary function $f$ in $C$ we thus have

$$
L(f)=\int_{0}^{2 \pi} H\left(e^{i \theta}\right) f\left(e^{i \theta}\right) d \theta=2 \pi H(0) f(0)=0
$$

since $H(0)=0$, and we can conclude that $E=C$.

If, on the other hand, (9) does not hold, the functions $f_{m}(z)$ must have a common zero in $|z| \leq 1$ and only functions which vanish at this point can belong to $E$.

As an immediate consequence of theorem 4 we get the following result.

Theorem 5. If (9) holds, then for every $g \in C$; functions $p_{1}, p_{2}, \ldots, p_{n}$ in $C$ exist such that

$$
\sum_{\nu=1}^{n} p_{\nu}(z) f_{\nu}(z) \equiv g(z) .
$$

It is clearly sufficient to prove the theorem for $g(z) \equiv 1$. By the theorem above, polynomials $P_{y}(z)$ can be chosen such that $\|F+1\|<\frac{1}{2}$, where

$$
F(z)=\sum_{v=1}^{n} P_{v}(z) f_{v}(z)
$$

In particular, $|F(z)|>\frac{1}{2}$ in $|z|<1$. We see that our relation is satisfied if we choose $p_{v}(z)=P_{v}(z) / F(z)$.

For later applications, we observe that the result holds for an arbitrary simply connected domain bounded by a Jordan curve - the analytic function which maps such a domain onto the unit circle is continuous on the boundary - and also that even in this more general case, polynomials $P_{v}(z)$ exist such that $\left|\sum_{1}^{n} P_{v}(z) f_{v}(z)\right| \geq \delta>0$ - this follows from a known approximation theorem of Walsh.

5. We shall now use theorem $\dot{5}$ to prove an analogous result for the space $B$. We must in this case replace condition (9) by a stronger assumption and we introduce the following notation. For a given function $f$ in $B$ and an arbitrary $a$ in $|a| \leq 1$, let us use the notation

$$
\mu_{f}(a)=\lim _{z \rightarrow a}|f(z)|
$$

where, for $|a|=1$, we have to approach $a$ from inside the unit circle.

Theorem 6. If $E$ is a subfamily of $B$ such that for every $a,|a| \leq 1, f \in E$ exists such that $\mu_{f}(a) \neq 0$, then any function $g$ in $B$ has a representation (10), where $f_{\nu}$ belongs to $E$ and $p_{\nu}$ belongs to $B$.

For every $a,|a|=1$, there is closed interval $A$ around $a$ and a function $f$ in $E$ such that $\mu_{f}(\zeta)>0$ for $\zeta \in A$. We cover $|z|=1$ by a finite number of these intervals $A_{1}, A_{2}, \ldots, A_{m}, A_{v}=\left(e^{i \alpha_{v}}, e^{i \beta_{v}}\right)$, and assume that 
L. CARLESON, On bounded analytic functions and closure problems

$$
0<\alpha_{1}<\beta_{m}<\alpha_{2}<\beta_{1}<\alpha_{3}<\beta_{2}<\ldots
$$

$(\bmod 2 \pi)$

Let $f_{v}$ be the function in $E$ which corresponds to $A_{v}$.

Since $\left|f_{v}(z)\right| \geq \delta>0$ in a neighbourhood of $A_{\nu}$, there are functions $w_{v}$ in $B$ such that $g_{v}(z)=w_{v}(z) \cdot f_{v}(z)$ are analytic and $\neq 0$ on $A_{v}$. Let us now choose a number $\gamma$ so that $\alpha_{2}<\gamma<\beta_{1}$. We construct a function $q_{1}(z) \in C$ such that

$$
\left\{\begin{array}{l}
\left|q_{1}\left(e^{i \theta}\right)\right|=1 \text { on } \alpha_{1} \leq \theta \leq \gamma \\
\left|q_{1}\left(e^{i \theta}\right)\right| \leq 1 \text { everywhere } \\
\left|q_{1}\left(e^{i \theta}\right)\right| \leq \varepsilon \text { on } \theta \leq \alpha_{1}-\varepsilon \text { and } \theta \geq \beta_{1} \quad \cdot(\bmod 2 \pi),
\end{array}\right.
$$

where $\varepsilon>0$ will be determined later. We may furthermore assume that $q_{1}(z) \neq 0$. A similar function $q_{2}(z)$ is constructed for $A_{2}$ with $\left|q_{2}\left(e^{i \theta}\right)\right|=1$ on $\left(\gamma, \beta_{2}\right)$. We next consider the functions

$$
\begin{aligned}
& F_{1}(z) \\
& F_{2}(z)
\end{aligned}=q_{1}(z) \cdot g_{1}(z) \pm q_{2}(z) \cdot g_{2}(z) .
$$

For $\varepsilon$ sufficiently small we obviously have $\mu_{F_{1}}(\zeta) \neq 0$ on $\left(\alpha_{1}, \alpha_{2}\right)$ and on $\left(\beta_{1}, \beta_{2}\right)$ and similarly for $F_{2}$. On the rest of the interval $\left(\alpha_{1}, \beta_{2}\right)$, i.e. on $\left(\alpha_{2}, \beta_{1}\right), F_{1}$ and $F_{2}$ are continuous and have no common zero, since such a zero would be a zero for $F_{1} \pm F_{2}$. We can as before multiply $F_{i}$ by a function $H_{i}$, which belongs to $B$, so that $G_{i}(z)=F_{i}(z) H_{i}(z), i=1,2$, is continuous on $\left(\alpha_{1}, \beta_{2}\right)$. We may also assume that $H_{i}(z) \neq 0$. The new functions $G_{i}$ are continuous in a domain $D: r_{0} \leq r \leq 1, \alpha_{1} \leq \arg z \leq \beta_{2}$ and in $D$ we have

$$
\left|G_{1}(z)\right|+\left|G_{2}(z)\right| \neq 0 \text {. }
$$

By the remark of theorem 5 , polynomials $P_{1}$ and $P_{2}$ exist such that

$$
\varphi_{1}(z)=P_{1}(z) \cdot G_{1}(z)+P_{2}(z) \cdot G_{2}(z)
$$

does not vanish on $\left(\alpha_{1}, \beta_{2}\right)$.

In our original situation we can thus use $\varphi_{1}$ instead of $f_{1}$ and $f_{2}$, and $A_{1}^{\prime}$ instead of $A_{1}$ and $A_{2}$. We continue the same process, which only consists in the forming of linear combinations, and we finally obtain a function $\varphi \in C$ which is of the form (10) and does not vanish for $|z| \geq \varrho$.

In the same way as above, we construct a function $\psi$ of the form (10) such that $\psi(z) \neq 0$ in $|z| \leq \varrho^{\prime}, \varrho^{\prime}>\varrho$. Finally, we consider the functions

$$
\begin{aligned}
& \phi_{1}(z) \\
& \phi_{2}(z)
\end{aligned}=\psi(z) \pm K \varphi(z) .
$$

If the constant $K$ is sufficiently large, then $\mu_{\phi_{i}}(a) \neq 0$ for $|a| \geq \varrho$, and we see as before that $\phi_{1}$ and $\phi_{2}$ have no common zeros. After multiplication by suitable, non-vanishing functions in $B$, we obtain two functions $\psi_{i}(z)$ in $C$ which are of the form (10) and have no common zeros. With the aid of these functions, we get by theorem 5 a linear representation of any function in $B$. The proof of theorem 6 is thus complete. 
6. As an illustration of the function-theoretic significance of theorem 6 , we make an application to the Pick-Nevanlinna interpolation problem.

Let $S=\left\{a_{v}\right\}$ be an infinite sequence in $|z|<1$. It is well-known that if there exists an analytic function $F(z)$ in $|z|<1$ such that

$$
\int_{0}^{2 \pi+} \log \left|F\left(r e^{i \theta}\right)\right| d \theta=O(1), \quad r \rightarrow 1,
$$

which vanishes on $S$ without vanishing identically, then there exists a function of the same kind in $B$; the condition on $S$ is given by

$$
\sum_{1}^{\infty}\left(1-\left|a_{\nu}\right|\right)<\infty
$$

We can then ask the following more general question: given a function $F$ and a set $S$, when does there exist a bounded function which takes the same values as $F$ on $S$ ? Unless $F$ is bounded, it is evidently necessary that (12) converge. We must also introduce some condition which ensures that $F$ is bounded on $S$. We shall here prove the following theorem. .

Theorem 7. Let $S$ be a given set such that (12) holds and suppose that arg $a_{v}$ belong to a closed set $E$. If the function $F(z)$ satisfies (11) and if furthermore

$$
\varlimsup_{z \rightarrow e^{i \theta}}|F(z)|<\infty, \quad \theta \in E,
$$

then the interpolation $f\left(a_{v}\right)=F\left(a_{v}\right), f \in B$, is possible.

$F(z)$ can be represented as the quotient of two bounded functions $\varphi(z)$ and $\psi(z)$, where $\psi(z)$ has no zeros. We may furthermore assume that $\mu_{\psi}(\zeta)>0$ on $E$, since $F(z)$ is bounded in a neighbourhood of $E$. If now

$$
\pi(z)=z \prod_{1}^{\infty} \frac{a_{v}-z}{1-z \vec{a}_{v}} \frac{\bar{a}_{v}}{\left|a_{v}\right|}, a_{\nu} \neq 0,
$$

then $\mu_{\psi}+\mu_{\pi} \neq 0$ in $|a| \leq 1$. By theorem 6 , functions $p$ and $q$ belonging to $B$ exist such that

$$
p(z) \psi(z)+q(z) \pi(z) \equiv \varphi(z)
$$

For $z=a_{v}$ we have

$$
p\left(a_{v}\right)=\frac{\varphi\left(a_{v}\right)}{\psi\left(a_{v}\right)}=F\left(a_{v}\right)
$$

$p(z)$ is hence a solution of the interpolation problem. 